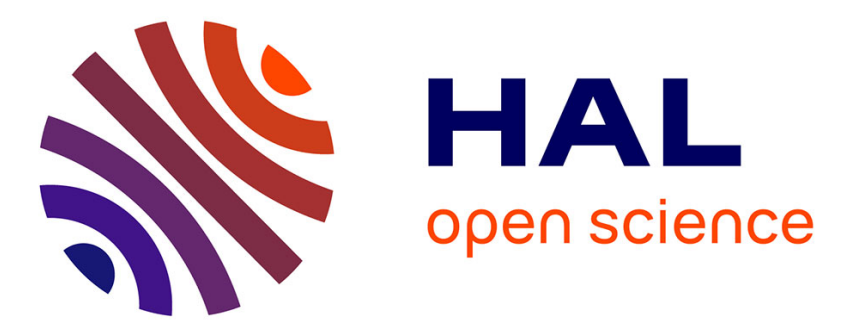

\title{
Validation de la traduction française d'un questionnaire court de dépression de Beck (BDI-FS-Fr)
}

\author{
Muaweah Alsaleh, Romain Lebreuilly
}

\section{To cite this version:}

Muaweah Alsaleh, Romain Lebreuilly. Validation de la traduction française d'un questionnaire court de dépression de Beck (BDI-FS-Fr). Annales Médico-Psychologiques, Revue Psychiatrique, 2017, 175 (7), pp.608-616. 10.1016/j.amp.2016.06.015 . hal-02313708

\section{HAL Id: hal-02313708 \\ https://hal.univ-lorraine.fr/hal-02313708}

Submitted on 11 Oct 2019

HAL is a multi-disciplinary open access archive for the deposit and dissemination of scientific research documents, whether they are published or not. The documents may come from teaching and research institutions in France or abroad, or from public or private research centers.
L'archive ouverte pluridisciplinaire HAL, est destinée au dépôt et à la diffusion de documents scientifiques de niveau recherche, publiés ou non, émanant des établissements d'enseignement et de recherche français ou étrangers, des laboratoires publics ou privés. 


\title{
Validation française d'un questionnaire court BDI-FS-Fr de dépression de Beck sur une population d'étudiants.
}

\author{
ALSALEH, Muaweah \\ Chercheur en psychologie, Centre d'Etude et de Recherche sur les Risques et les \\ Vulnérabilités (CERReV), EA 3919, Université de Caen Normandie, Esplanade de la Paix, \\ 14032 Caen Cedex, France.

\section{LEBREUILLY, Romain} \\ Maitre de conférences en Psychologie, Laboratoire InterPsy (EA 4432), groupe GRC, \\ Nancy
}




\section{Résumé}

Objectif : Cet article présente la validation française de l'Inventaire de BDI-FS (Beck Depression Inventory-Fast Screen) sur un échantillon d'étudiants.

Méthode : Une étude transversale a été menée sur 473 étudiants de l'Université de Caen Normandie.

Résultats : Le BDI-FS-Fr (Beck Depression Inventory-Fast Screen-France) obtient une bonne sensibilité, spécificité et une bonne valeur prédictive positive et négative, un coefficient de Yule très fort et une aire sous la courbe satisfaisante. Il a une très bonne validité de structure interne : une analyse factorielle exploratoire a dégagé un facteur expliquant $40,21 \%$ de la variance totale du BDI-FS-Fr, et il présente une consistance interne satisfaisante $(0,74)$. Le BDI-FS-Fr a une très bonne validité de structure externe (validité de construit : validité convergente et divergente; $\mathrm{p}<0,001)$ et discriminante $(\mathrm{p}<0,001)$, et validité concomitante $(\mathrm{p}<0.001))$. Le coefficient de fidélité pair-impair est à $0.73(\mathrm{p}<0,001)$. La fidélité test-retest est de $0,82(\mathrm{p}<0,001)$.

Conclusion : La version française du BDI-FS possède des propriétés psychométriques très favorables. Elle est valide pour auto-évaluer la dépression, son intensité et son évolution, et elle est un bon complément au BDI pour les étudiants. Le BDI-FS-Fr présente ainsi de très bonnes qualités psychométriques et constitue un outil utile pour les chercheurs et les praticiens.

Mots clés : Dépression, BDI-FS-Fr, Fidélité, validité, questionnaire, psychométrique, étudiants. 


\section{Introduction}

La dépression est reconnue comme un problème de santé majeur [16]. Selon le rapport de l'Organisation mondiale de la santé (OMS), la maladie mentale est répandue dans toutes les couches de la société $[39,22]$ et la dépression peut aussi s'accompagner de symptômes somatiques [17]. La dépression, l'anxiété et le stress sont les principales pathologies chez des personnes vivant avec un handicap. L'impact de la dépression non traitée et de l'anxiété sur la capacité à bien vivre est égal ou supérieur à celui d'autres problèmes de santé courants tels que les maladies cardiaques ou l'arthrite [30, 22]. Plusieurs études ont montré que l'humeur dépressive altérait l'estime de soi $[10,11,31]$.

Gustavsson et al., en 2011 estiment que les maladies dépressives sont les maladies psychiatriques les plus coûteuses en Europe mais aussi dans le monde étant donné leur forte prévalence, leur survenue précoce, leur chronicité et les handicaps qui s'ensuivent [1]. Selon les estimations de l'OMS en 2012, la dépression touche plus de 350 millions de personnes et c'est la première cause d'incapacité dans le monde. Le nombre de décès par suicide suite à une dépression sévère est estimé à plus d'un million par an dans le monde [2].

La prévalence annuelle établie par la recherche épidémiologique est de 6,9\% en Europe [3] et de 6,6\% aux Etats-Unis [4,5]. La prévalence annuelle est faible chez les enfants quelque soit leur sexe (environ 1\%), elle atteint $8 \%$ chez les adolescents, avec une augmentation plus forte chez les filles [6,7]. En France, la prévalence annuelle de la dépression est estimée à $8 \%$ chez les 18-75 ans. Les femmes sont deux fois plus touchées que les hommes [8]. Les troubles dépressifs atteignent une personne sur cinq au cours de sa vie, dont $21 \%$ de femmes et $13 \%$ d'hommes [9]. La prévalence de la dépression chez des étudiants dans la période des études est estimée entre $27,1 \%$ [12] et 37,2\% [13].

La littérature scientifique fournit un grand nombre d'outils validés pour évaluer la gravité du trouble dépressif comme l'échelle de dépression de Hamilton [14,15]; l'inventaire de dépression de Beck - 2e édition (BDI-21) [14] ; l'échelle de dépression de Montgomery et Asberg (MADRS) [14-18] et le questionnaire sur la santé du patient-9 (Patient Health Questionnaire - PHQ-9) [15,19].

Pour évaluer et identifier la dépression, l'outil d'auto-évaluation le plus répandu est la « Beck Depression Inventory-II » (BDI-II) [21]. En utilisant le BDI-II en tant que base, le BDIFS a été développé pour factoriser des signes neurovégétatifs de dépression trouvés chez les patients et se concentrer sur les facteurs de la dépression qui se rapportent uniquement à la dépression [23]. Beck a étendu ses recherches sur cette mesure (BDI-FS) pour inclure les patients à partir des pratiques familiales, des pratiques de médecine interne, des pratiques pédiatriques et des consultations [24]. Le but de la présente étude a été de valider la version anglaise de l'échelle d'auto-évaluation de la dépression de BDI-FS (Beck Depression Inventory-Fast Screen), d'étudier si elle conserve ses propriétés psychométriques dans sa version française et de valider le BDI-FS-Fr comme un outil de dépistage et aussi de diagnostic de la dépression chez les étudiants. Cette étude évalue ces propriétés en les comparant notamment à celles de l'échelle d'auto-évaluation de Beck Depression Inventory (BDI-II) en français.

\section{Méthode}

\section{1. Échantillon}

L'échantillon est composé de 473 étudiants (77 hommes et 396 femmes) volontaires de l'Université de Caen âgés de 17 à 24 ans (âge : $M=18.66$, ET =1.26). La participation est volontaire. 


\subsection{Procédure}

Cette étude a reçu l'approbation de la Maison des Recherches de Sciences Humaines et du laboratoire (MRSH et CERReV) de l'Université Caen Normandie. Les participants sont recrutés pendant leur enseignement du premier semestre de l'année universitaire. La recherche était présentée comme une étude sur les troubles émotionnels (dépression, anxiété et stress), et sur l'estime de soi des étudiants. Les participants étaient invités à remplir les questionnaires de BDI-FS-Fr, de BDI-II, d'EDAS-21, et d'estime de soi (EDSR) de façon anonyme.

\subsection{Analyse statistique}

Les propriétés psychométriques étudiées sont la fiabilité de l'outil, qui comprend notamment la cohérence interne et la validité de critère et de construit. Dans un premier temps, nous avons calculé la moyenne (M), et l'écart-type (ET), pour toutes les données. Dans un deuxième temps, l'adéquation des items et la consistance interne de l'échelle ont été estimées par le coefficient alpha de Cronbach qui constitue chaque dimension de l'outil [40]. La cohérence interne d'une dimension est considérée comme bonne quand le coefficient est supérieur à $0,70[35 ; 42 ; 41]$. Dans un troisième temps, la validité de critère d'une échelle a été estimée par le coefficient de corrélation des rangs de Spearman. La validité de critère d'une échelle est la conformité de son résultat avec ceux de mesures plus traditionnelles ou ceux d'autres instruments validés dans le même domaine [36]. Celle-ci a été étudiée par la mesure des corrélations existant entre les scores des dimensions et l'évaluation de la dépression, par le calcul du coefficient de corrélation des rangs de Spearman. La validité convergente et divergente a été établie au moyen des mesures d'inter-corrélations (via le $r$ de Bravais-Pearson) entre le score BDI-FS-Fr, BDI-II, EDAS-21 et EDSR. En accord avec la définition de la validité convergente formulée par Smith et al. (2005) [43], l'hypothèse stipule que cette qualité psychométrique sera démontrée si les valeurs des corrélations entre les variables et les différents scores des échelles sont significatives et nous le supposons. Dans un quatrième temps, les valeurs diagnostiques ont été calculées : sensibilité, spécificité, valeurs prédictives positives et négatives, rapport des cotes, la courbe ROC (Receiver Operating Characteristic) et l'aire sous la courbe. Les notions de sensibilité et de spécificité décrivent les propriétés intrinsèques d'un test, et la valeur prédictive positive (VPP) et la valeur prédictive négative (VPN) indiquent la probabilité de porter, ou non, la maladie recherchée par le test, en fonction de son résultat positif ou négatif [44 ; 49]. La courbe ROC permet d'étudier les variations de la spécificité et de la sensibilité d'un test pour différentes valeurs du seuil de discrimination [53]. L'aire sous la courbe ROC est un index simple et quantitatif de la performance d'un test [47]. Dans un cinquième temps, la fidélité test-retest ${ }^{1}$ concerne donc la reproductibilité de l'outil. Elle a été évaluée en calculant la corrélation entre les résultats de deux passations du BDI-FS-Fr. Les étudiants ont d'abord complété le BDI-FS-Fr et ensuite complété un second exemplaire du questionnaire 12 jours plus tard car les étudiants ne devaient pas prendre connaissance du premier questionnaire lorsqu'ils remplissaient le second. Enfin, les données du BDI-FS-Fr ont aussi été soumises à une analyse factorielle pour vérifier la validité de structure.

L'analyse statistique a été effectuée en utilisant un logiciel informatique $\mathrm{R}$ (langage de programmation, version R3.2.0).

\subsection{Mesures et Instrument}

\subsubsection{Le BDI-II (Beck Depression Inventory-II)}

La dépression a été évaluée en utilisant l'Inventaire de Dépression de Beck dans sa version à 21 items (BDI-II), [21] qui est un auto-questionnaire d'évaluation composé de 21 groupes d'énoncés et qui évalue l'état de l'humeur au cours des deux dernières semaines. Le BDI-II est une version mise à jour du BDI original et donne une image plus précise des critères de la

\footnotetext{
${ }^{1}$ «La fidélité test-retest d'un instrument est sa capacité à reproduire les mêmes résultats lors de deux administrations différentes » [71].
} 
dépression dans le Manuel Diagnostique et Statistique des Troubles-IV-TR mentaux (DSMIV-TR) [25]. L'inventaire de dépression de Beck (BDI-II; Beck et al., 1996 ; adaptation française par Pichot et al.,) [50] est un questionnaire d'auto-évaluation mesurant la sévérité de la dépression composé de 21 items cotés de 0 à 3 (score total de 0 à 63) [50].

\subsubsection{Le BDI-FS (Beck Depression Inventory-FastScreen)}

Le BDI-FS est une version courte de la BDI-II. Le BDI-FS [24] est composé de 7 items d'auto-évaluation pris à partir des 21 items de l'Inventaire de Dépression de Beck (BDI-II) [29]. Le BDI-II a déjà été traduit et validé en français [69, 70]. En 2000, Beck et al., ont validé une version courte anglaise du BDI-II qui inclut 7 items. Les propriétés psychométriques du BDI-FS demandent à être évaluées en français. Notre version du BDI-FS (française) est constituée des mêmes items que la version de Beck et al., 2000. Les 7 items du BDI-FS français sont extraits de la version française du BDI-II (Annexe 1).

Pour développer les 7 items du BDI-FS, Beck a combiné les deux critères diagnostiques du DSM-IV-TR (anhédonie «anhedonia » et de tristesse «sadness »), ainsi qu'un critère de suicidalité « suicidality ${ }^{2}$ » et les quatre composantes cognitives ${ }^{3}$ dérivées du BDI-II qui constituaient l'un des facteurs de la BDI-II (Beck et al., 2000). Les items somatiques sont exclus pour augmenter la spécificité pour les patients. Ces sept items composent la structure interne de la BDI-FS.

Les items sont mis en place pour évaluer l'état de l'humeur lors des 2 dernières semaines sur l'échelle de Likert à 4 points (0-3), donnant un score total maximum de 21 . La valeur de seuil (score optimal) du BDI-FS $\geq 4$ avait une sensibilité élevée $(1,00)$ et une spécificité $(0,84)$ [31]. Ce seuil optimal est cohérent avec les études précédentes rapportées dans le manuel de test [24]. Les sept éléments spécifiques sur cette mesure comprennent la tristesse, le pessimisme, le sentiment d'échec dans le passé, la perte de plaisir, la baisse d'estime de soi, l'autocritique et des pensées suicidaires ${ }^{4}$ [23, 24]. Lorsque Beck et al. (1997) ont comparé le BDI-FS avec une autre mesure standardisée de la dépression, le BDI-FS était positivement corrélé à 0,62, p<0,001 démontrant ainsi une validité convergente notable [34]. Cette mesure prend environ 2 minutes.

\subsubsection{L'échelle de dépression, d'anxiété et de stress (EDAS-21)}

Ce questionnaire développé par Lovibond et Lovibond [20] mesure l'anxiété, la dépression et le stress. Il comprend 21 questions réparties en trois échelles ( 7 items pour chaque échelle) : la dépression (par exemple : " j'ai eu l'impression de ne pas pouvoir ressentir d'émotion positive »), l'anxiété (par exemple : "j'ai été conscient(e) d'avoir la bouche sèche ») et le stress (par exemple : "j'ai trouvé difficile de décompresser»). L'échelle de réponses est de type Likert en 4 points allant de 0 signifiant « ne s'applique pas du tout à moi », jusqu'à 3 signifiant «s'applique entièrement à moi, ou la grande majorité du temps ». Les scores s'étendent ainsi de 21 à 84. La traduction a été effectuée par l'équipe de Martin à l'université d'Ottawa, elle est disponible sur un site web dédié au DASS [26]. Les coefficients de consistance interne des trois différentes sous-échelles de l'échelle de dépression, d'anxiété et de stress-21 (EDAS-21) de la version francophone du DASS-21 varient entre 0,89 et 0,94. La version francophone présente aussi une bonne validité convergente et discriminante.

\subsubsection{Le questionnaire d'Estime De Soi de Rosenberg (EDSR)}

Le questionnaire d'estime de soi de Rosenberg (Le Rosenberg Self-Esteem Scale « RSE ») [27] est l'outil le plus fréquemment employé, du fait notamment de sa validité bien établie [37]. Il comporte 10 items évalués sur une échelle de type Likert en 4 points, allant de $1=$ tout à fait d'accord à $4=$ pas du tout d'accord. Le questionnaire présente une validité de construit ainsi qu'une fidélité temporelle satisfaisante $(\alpha=0,85)$ [33]. Schmitt et Allik en 2005 [28]

\footnotetext{
${ }^{2}$ Compte tenu du caractère impératif d'identifier les facteurs de risque de suicide, la tendance suicidaire est évaluée dans cette mesure et constitue ainsi un item du BDI-FS.

${ }^{3}$ Pessimism, past failure, self-dislike and self-criticalness

${ }^{4}$ Sadness, Pessimism, Past Failure, Loss of Pleasure, Self-Dislike, Self-Criticalness, Suicidal Thoughts.
} 
rapportent un coefficient Alpha de Cronbach moyen de 0,81, obtenu sur un total de 16998 personnes réparties dans 53 pays. Le score varie de 10 à 40, plus le score est élevé plus l'estime de soi est élevée. Un score inférieur à 25 montre une estime de soi faible. La version française d'EDSR a été validée par Vallières et Vallerand en 1990 [38].

\section{Résultats}

\subsection{Analyses préliminaires : statistiques descriptives}

Nous avons réalisé une première phase d'analyse descriptive dont les résultats sont présentés dans le Tableau 1. Les caractéristiques démographiques des étudiants sont décrites à l'aide des moyennes, écart-types et médianes. Les 473 étudiants inclus dans l'étude étaient 77 hommes et 396 femmes d'âge moyen 18.66 ans (écart-type=1.26).

Tableau 1 : Analyses Statistiques descriptives.

\begin{tabular}{|l|c|c|}
\hline Variable & M & ET \\
\hline Age & 18.66 & 1.26 \\
\hline Sexe $-\mathrm{n}(\%)$ & \multicolumn{2}{|c|}{$77(16.28 \%)$} \\
\hline Hommes & \multicolumn{2}{|c|}{$396(83.72 \%)$} \\
\hline Femmes & 8.32 & 6.40 \\
\hline Dépression-BDI-II & 3.80 & 2.95 \\
\hline Dépression-BDI-FS-Fr & 4.27 & 3.98 \\
\hline Dépression-EDAS-21 & 4.90 & 4.35 \\
\hline Anxiété-EDAS-21 & 6.42 & 5.09 \\
\hline Stress-EDAS-21 & 30.06 & 5.50 \\
\hline Estime de soi (EDSR) & \multicolumn{2}{|}{} \\
\hline Nombre (N) : 473 & \multicolumn{2}{|c|}{} \\
\hline
\end{tabular}

\subsection{Applicabilité}

La durée de passation pour le BDI-FS-Fr est de +/- 2 minutes. L'objectif du questionnaire est très bien compris par les étudiants : ils s'accommodent très vite aux consignes de passation et il n'y a eu aucun problème de compréhension des items. Les examinateurs n'ont observé aucune demande d'interruption partielle (pause), ni totale (arrêt) lors de la passation du questionnaire. Il faut aussi préciser que nous n'avons aucune donnée manquante, car l'étudiant répondait au BDI-FS-Fr en présence d'un examinateur qui s'assurait que tous les items étaient bien compris et complétés.

\subsection{Validité de construit}

\subsubsection{Validité convergente et divergente}

La validité de structure externe a été explorée par la recherche de la validité convergente et divergente [56, 57, 52]. Les relations entre BDI-FS-Fr, BDI-II, D-EDAS-21, A-EDAS-21 et S-EDAS-21 (validité convergente), entre BDI-FS-Fr et EDSR (validité divergente) ont été testées à l'aide de corrélations $\mathrm{r}$ de Pearson. Le tableau 2 reprend les inter-corrélations entre les scores de dépression, d'anxiété, de stress et d'estime de soi. Le BDI-FS-Fr est corrélé de manière significative avec les scores de dépression ( $\mathrm{r}=0,53$ et $0.57 ; \mathrm{p}<0,001)$, d'anxiété $(\mathrm{r}=0,42 ; \mathrm{p}<0,001)$ et de stress $(\mathrm{r}=0,42 ; \mathrm{p}<0,001)$, et de manière significative et négative avec le score d'estime de soi $(\mathrm{r}=-0,54 ; \mathrm{p}<0,001)$. Ces corrélations confirment la validité convergente et divergente du BDI-FS-Fr. Donc, le BDI-FS-Fr a une bonne validité de construit (validité convergente et divergente).

Les corrélations entre le BDI-FS-Fr et les échelles de DEP et l'EDSR ont une taille d'effet importante selon Cohen mais les corrélations entre le BDI-FS-Fr et les échelles d'ANX (anxiété) et du STR (stress) ont une taille d'effet modérée (Cohen, 1992). 
Tableau 2 : Les inter-corrélations entre les scores de dépression, d'anxiété, de stress et d'estime de soi.

\begin{tabular}{|c|c|c|c|c|c|c|}
\hline questionnaires & $\begin{array}{l}\text { BDI- } \\
\text { II }\end{array}$ & BDI-FS & EDSR & $\begin{array}{c}\text { D- } \\
\text { EDAS }\end{array}$ & $\begin{array}{c}\text { A- } \\
\text { EDAS }\end{array}$ & $\begin{array}{c}\text { S- } \\
\text { EDAS }\end{array}$ \\
\hline $\begin{array}{l}\text { Dépression- } \\
\text { BDI-II }\end{array}$ & 1.00 & $0.53 * * *$ & $0.63^{-} * *$ & $0.70 * * *$ & $0.63 * * *$ & $0.56^{* * *}$ \\
\hline $\begin{array}{l}\text { Dépression - } \\
\text { BDI-FS-Fr }\end{array}$ & 0.52 & 1.00 & $\begin{array}{c}- \\
0.54 * * *\end{array}$ & $0.57 * * *$ & $0.42 * * *$ & $0.42 * * *$ \\
\hline $\begin{array}{l}\text { Estime de soi- } \\
\text { EDSR }\end{array}$ & -0.63 & -0.54 & 1.00 & $\begin{array}{c}- \\
0.60 * * *\end{array}$ & $\stackrel{-}{-}$ & $\begin{array}{c}- \\
0.42 * * *\end{array}$ \\
\hline $\begin{array}{l}\text { Dépression- } \\
\text { EDAS-21 }\end{array}$ & 0.70 & 0.57 & -0.60 & 1.00 & $0.66 * * *$ & $0.63 * * *$ \\
\hline $\begin{array}{l}\text { Anxiété- } \\
\text { EDAS-21 }\end{array}$ & 0.62 & 0.42 & -0.45 & 0.66 & 1.00 & $0.75^{* * *}$ \\
\hline $\begin{array}{l}\text { Stress- } \\
\text { EDAS-21 }\end{array}$ & 0.55 & 0.42 & -0.42 & 0.63 & 0.75 & 1.00 \\
\hline
\end{tabular}

\subsubsection{Validité discriminante}

Le résultat montre que le BDI-FS-Fr distingue les groupes déprimés et non déprimés $(\mathrm{t}=$ 15.029 , df $=256$, p-value $<0,00001$ ), ainsi le questionnaire du BDI-FS-Fr est valide auprès d'une population d'étudiants.

En résumé, la validité du construit du questionnaire du BDI-FS-Fr a été vérifiée en utilisant la validité convergente, divergente et discriminante. Le résultat montre que le questionnaire du BDI-FS-Fr a une très bonne validité du construit car les trois validités sont significatives $(0,001 ; 0,01 ; 0,05)$. Le questionnaire du BDI-FS-Fr jouit également d'une bonne validité de structure externe.

\subsection{Validité contre critère ${ }^{5}$}

Le phénomène mesuré est évalué à la fois par le questionnaire étudié et par un critère extérieur à celui-ci qui est pris comme norme de référence ${ }^{6}[59]$.

\subsubsection{Validité concomitante ${ }^{7}$}

Une analyse corrélationnelle a été menée à l'aide du coefficient de corrélation des rangs de Spearman entre le BDI-FS-Fr et toutes les variables pour réaliser la validité de critère d'une échelle [36]. Le BDI-FS-Fr était significativement et positivement corrélé au BDI-II, rhô $=0,78, \mathrm{p}<0,001$, à l'EDAS-21, rhô $=0,65 ; 0,52 ; 0,49, \mathrm{p}<0,001$ et négativement corrélé à l'EDSR, rhô $=-0,64, \mathrm{p}<0,001$ (Tableau 3). Enfin, ce questionnaire montrait une bonne validité concomitante avec l'échelle BDI-II, EDAS-21.

\footnotetext{
${ }^{5}$ Ou la validité de critère.

${ }^{6}$ Gold Standard.

${ }^{7}$ Lorsque le critère de référence est utilisé en même temps que la passation du test «l'échelle » chez chaque sujet «par exemple le même jour », nous parlerons de validité concourante ou concomitante $[85,59]$.
} 
Tableau 3 : Corrélations de Spearman

\begin{tabular}{|c|c|c|c|}
\hline Variable & $\begin{array}{c}\text { Coefficient de corrélation des } \\
\text { rangs de Spearman (rhô), BDI-FS- } \\
\text { Fr }\end{array}$ & $\begin{array}{c}\text { Significativit } \\
\text { é (valeur-p) }\end{array}$ & $\begin{array}{c}\text { Intervalle } \\
\text { de confiance }\end{array}$ \\
\hline Dépression-BDI-II & 0,79 & 0,000 & $0.74-0.83$ \\
\hline $\begin{array}{l}\text { Dépression- EDAS- } \\
21\end{array}$ & 0,65 & 0,000 & $0.59-0.71$ \\
\hline Anxiété- EDAS-21 & 0,52 & 0,000 & $0.45-0.59$ \\
\hline Stress- EDAS-21 & 0,49 & 0,000 & $0.42-0.56$ \\
\hline $\begin{array}{l}\text { Estime de soi } \\
\text { (EDSR) }\end{array}$ & $-0,64$ & 0,000 & $-0.58-$ \\
\hline
\end{tabular}

3.4.2. Analyse de la sensibilité et de la spécificité du BDI-FS-Fr avec les échelles de dépression (test de référence, BDI-II et EDAS-21)

473 étudiants ont répondu aux BDI-FS-Fr, BDI-II et EDAS-21. La sensibilité du BDI-FSFr en comparaison avec BDI-II et EDAS-21 signifie que lorsque la personne est dépressive, il y a plus de $71 \%$ de possibilités que le test de dépistage de la dépression soit positif. La spécificité de ce questionnaire indique que lorsque la personne n'est pas dépressive, il y a plus de $82 \%$ de possibilités que le test de dépistage de la dépression soit négatif. Dans notre recherche, la sensibilité et la spécificité montrent que le questionnaire du BDI-FS-Fr semble fortement fiable.

Concernant les valeurs de VPP et de VPN, la VPP du BDI-FS-Fr en comparaison avec BDI-II et EDAS-21signifie que la personne a plus de $62 \%$ de possibilités de dépression quand le test de dépistage de la dépression est positif. Sa VPN signifie que la personne a plus de $87 \%$ de possibilités de ne pas être dépressive quand le test de dépistage de la dépression est négatif (Tableau 4). D'après les résultats de cette étude, le rapport de vraisemblance positif $(\mathrm{RV}+)$ indique qu'il y a quatre fois plus de possibilités de présenter un test positif sur le BDIFS-Fr chez les étudiants lorsque la personne est dépressive que lorsqu'elle ne l'est pas. Le rapport de vraisemblance négatif (RV-) indique qu'il y a trois fois plus de possibilités de présenter un test négatif sur le BDI-FS-Fr chez les étudiants lorsque la personne n'est pas dépressive que lorsque la personne est dépressive. Selon le critère du RV (plus RV+ est élevé et plus RV- est proche de 0, plus un test est efficace, Bewick et al., 2004 [45]), les valeurs du rapport de vraisemblance positif $(\mathrm{RV}+)$ et négatif $(\mathrm{RV}-)$ indiquent que le BDI-FS-Fr est efficace (Tableau 4).

Pour estimer l'efficacité et l'aspect discriminatoire de BDI-FS-Fr, nous avons également utilisé d'autres critères. Ainsi, selon le critère de RC (Rapport des Cotes) de Cucherat en 2009 [48], (plus le RC est éloigné de 1, plus l'effet est important). Les résultats ont montré un bon rapport des cotes $(\mathrm{RC}=18.359$ et $11.414>1)$ ce qui indique que le test est discriminatoire. De plus, selon le coefficient Q de Yule [46], l'intensité de la liaison entre les deux variables (BDIFS-Fr et BDI-II ; BDI-FS-Fr et EDAS-21) est très forte (respectivement 0,90 et 0,84). Le résultat du test de la proportion de sujets bien classés (BC, l'efficacité diagnostique) a montré que $82.02 \%$ de l'échantillon est dépisté correctement par le BDI-FS-Fr en comparaison avec le BDI-II et que $80.12 \%$ de l'échantillon est dépisté correctement par le BDI-FS-Fr en comparaison avec l'EDAS-21(Tableau 4). 
Tableau 4 : Valeurs diagnostiques du BDI-FS-Fr

BDI-FS-Fr comparé avec BDI-II et EDAS-21

\begin{tabular}{|c|c|c|c|c|}
\hline & \multicolumn{2}{|c|}{ BDI-FS-Fr et BDI-II } & \multicolumn{2}{|c|}{ BDI-FS-FR et EDAS-21 } \\
\hline Résultat & & $\begin{array}{l}\text { IC: Intervalle } \\
\text { de confiance }(95 \\
\%)\end{array}$ & & $\begin{array}{l}\text { IC : Intervalle } \\
\text { de confiance }(95 \\
\%)\end{array}$ \\
\hline Sensibilité (SENS) & $78.17 \%$ & $\begin{array}{l}\text { IC }: 0,4533- \\
0,6152\end{array}$ & $71.63 \%$ & $\begin{array}{ccc}\text { IC: } & 0,6319 \quad- \\
0,7795 & \end{array}$ \\
\hline Spécificité (SPEC) & $83.68 \%$ & $\begin{array}{c}\text { IC : } 0,9209- \\
0,9684\end{array}$ & $82.25 \%$ & $\begin{array}{cc}\text { IC: } & 0,7782- \\
0,8595 & \end{array}$ \\
\hline Valeur prédictive positive (VPP) & $67.07 \%$ & & $62.73 \%$ & \\
\hline Valeur prédictive négative (VPN) & $89.97 \%$ & & $87.15 \%$ & \\
\hline Prévalence $(\mathrm{P})$ & $29.64 \%$ & & & \\
\hline Rapport des cotes (RC) & 18.359 & $\begin{array}{c}\text { IC }: 11.15- \\
29.93\end{array}$ & 11.414 & IC : $7.22-18.04$ \\
\hline $\begin{array}{l}\text { Rapport de vraisemblance positif } \\
(\mathrm{RV}+)\end{array}$ & 4.790 & $\begin{array}{ccc}\mathrm{IC} & : 3.704 & - \\
6.193 & & \\
\end{array}$ & 4.007 & $\begin{aligned} & \text { IC: } 3.113 \\
& 5.157 \\
&\end{aligned}$ \\
\hline $\begin{array}{l}\text { Rapport de vraisemblance négatif } \\
\text { (RV-) }\end{array}$ & 0.261 & 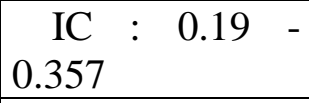 & 0.351 & IC: $0,27-0,457$ \\
\hline Coefficient Q de Yule (CQ) & 0.90 & & 0.84 & \\
\hline $\mathrm{BC}$ & $8202 \%$ & & $80.12 \%$ & \\
\hline
\end{tabular}

\subsubsection{L'analyse ROC (Receiver Operating Characteristic) et la capacité} discriminante

Plus la courbe ROC tend vers l'extrémité supérieure gauche du graphique, plus l'aire résultant sous cette courbe est élevée, meilleure est la capacité discriminante [51]. La courbe ROC du BDI-FS-Fr est illustrée à la figure 1. Le BDI-FS-Fr y démontre une bonne capacité à discriminer les sujets dépressifs des sujets sains, comme en témoigne l'estimation de l'aire sous la courbe (ASC) qui vaut 0.81 et 0,77 selon le test de référence. 
ROC

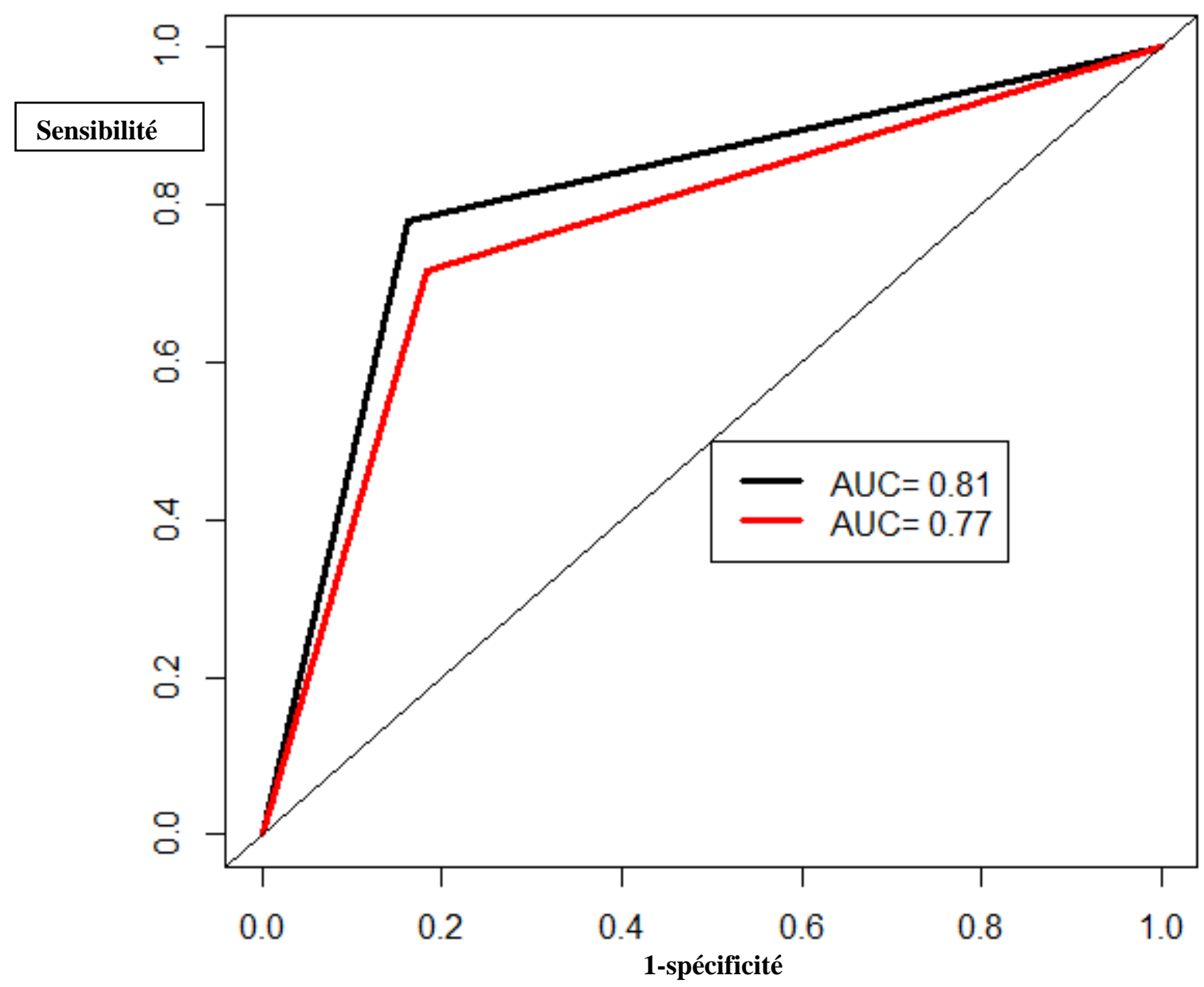

Figure 1 : Courbe ROC ${ }^{8}$ du BDI-FS-Fr comparée avec le BDI-II et l'EDAS-21 : sensibilité en fonction de 1spécificité. Le ROC indique que l'instrument est d'une grande utilité pour prédire la présence ou l'absence de la dépression.

\subsubsection{Aire sous la courbe (ASC)}

Pour un test parfait, l'aire sous la courbe vaut 1 . Pour un test sans valeur discriminante, l'aire sous la courbe vaut 0,5 [47 et 65]. L'aire sous la courbe ROC ou AUC (Area Under the Curve) est une estimation de l'efficacité globale du test [65]. Swets (1988) a défini une échelle d'interprétation de l'efficacité d'un test en fonction de la valeur de l'AUC [54 et 65]. Pour le test de BDI-FS-Fr chez les étudiants, l'aire sous la courbe vaut 0.81 (BDI-FS-Fr et BDI-II) et 0,77 (BDI-FS-Fr et EDAS-21) (intervalle de confiance à $95 \%$ ). Selon la valeur de l'AUC défini par Swets en 1988, l'efficacité du BDI-FS-Fr est satisfaisante car les valeurs de deux aires sous la courbe $(0.81 ; 0.77)$ se situent entre 0,70 et 0,90 (critère satisfaisant selon Swets [54]).

\subsubsection{Probabilité post-test (ou prévalence) de la maladie}

Nous pouvons déterminer la probabilité post-test de la dépression selon le théorème du Révérend Bayes [49], qui permet de déterminer la probabilité a posteriori d'une maladie en fonction du résultat du test, à partir des seules valeurs de probabilité a priori, sensibilité et spécificité du test [49].

\footnotetext{
${ }^{8}$ Selon Wasson et al., 2005, d'une part, la variable '1-spécificité' se situe sur l'axe des abscisses (égale à l'effectif de faux positifs parmi les groupes en bon état). D'autre part, la sensibilité (égale à l'effectif de vrais positifs parmi les groupes en mauvais état) se trouve sur l'axe des ordonnées [65].
} 
En appliquant ce théorème à notre étude, nous pouvons également déterminer la probabilité de dépression en fonction de diverses valeurs de probabilité pré-test. La probabilité post-test de l'échantillon, test positif ${ }^{9}$, est de 67\% (IC 95\% [61\%,73\%]) entre BDI-FS-Fr et BDI-II ; et de $62 \%$ (IC 95\% [56\%,68\%]) entre BDI-FS-Fr et EDAS-21. Ce résultat illustre l'importance de l'influence de la probabilité a priori sur la probabilité a posteriori. La probabilité pré-test de la dépression dans l'échantillon (prévalence) est très élevée (29.64\%), sa probabilité posttest est, un test négatif ${ }^{10}$, de $10 \%$ (IC 95\% [7\%,13\%]) et de 13\% (IC 95\% [10\%,16\%]) en l'occurrence. Ceci illustre bien l'importance de déterminer la probabilité a priori de la maladie chez un étudiant (probabilité pré-test de l'individu), avant d'effectuer un test, et d'anticiper ce que deviendra sa probabilité a posteriori en fonction du résultat du test. Ceci afin de comparer les probabilités pré- et post-test de l'échantillon d'une part et de l'individu d'autre part.

\subsection{Fidélité du BDI-FS-Fr}

Pour éprouver la fidélité et la validité de structure interne de l'échelle [56, 57] du BDI-FSFr nous avons procédé à deux types d'analyses : - une analyse des items où nous avons vérifié la distribution des réponses aux différentes questions, les corrélations entre questions et échelles, et l'homogénéité interne des échelles à l'aide de l'alpha de Cronbach [35]. - une analyse factorielle exploratoire (AFE) [59].

\subsubsection{Cohérence interne et fidélité du BDI-FS-Fr}

L'alpha de Cronbach [35] qui permet d'estimer la fidélité et de juger la cohérence interne des échelles montre que l'échelle du BDI-FS-Fr a une homogénéité très forte et élevée. Concernant la cohérence interne du BDI-FS-Fr, le coefficient alpha de Cronbach est supérieur à 0,70 . Le résultat obtenu par l'analyse de fiabilité qui mesure la consistance interne des items, est très bon ; il est de $\mathbf{0 , 7 4}$ pour le score BDI-FS-Fr. Concernant le sexe, la cohérence interne du BDI-FS-Fr chez les femmes et chez les hommes était bonne avec des coefficients alpha de Cronbach de $\mathbf{0 , 7 4}$ pour les deux sexes. L'estimation de la fiabilité était très bonne pour l'échelle du BDI-FS-Fr. Ce score suggère que les items du BDI-FS-Fr, tels qu'ils sont utilisés au sein d'un échantillon d'étudiants possèdent une cohérence interne relativement élevée.

Le coefficient de fidélité, effectué par Spearman-Brown, de la bissection (pair-impair) est à 0.73 ( $\mathrm{p}<0,0001$ ) et le coefficient de fidélité par moitiés (méthode "split-half", division de manière aléatoire en deux parties) est à $0,75(p<0,0001)$.

\subsubsection{Fidélité test-retest}

Parmi l'échantillon total, la fidélité test-retest ${ }^{11} \mathrm{du}$ BDI-FS-Fr a été effectuée en demandant aux 46 étudiants ( 35 femmes et 11 hommes) de compléter le questionnaire deux fois avec un intervalle de 12 jours. Le coefficient de corrélation de Pearson pour la fidélité test-retest est de 0,82 [IC 95\% : 0.69-0.89] ( $\mathrm{p}<0,0001)$. Ainsi, le BDI-FS-Fr est reproductible.

\section{Validité interne du BDI-FS-Fr}

\subsection{Validité factorielle et structure factorielle}

La validité de structure interne a été explorée notamment par les analyses en composantes principales (ACP) [56, 57]. C'est la méthode d'analyse factorielle la plus utilisée [58- 61]. Pour appréhender la structure du BDI-FS-Fr, nous avons effectué une ACP avec rotation varimax sur les 7 items.

\subsection{Analyse d'items}

La cohérence interne des items du BDI-FS-Fr a également été estimée en calculant la matrice de corrélation des items entre eux. La corrélation inter-items pour les 7 items du BDI-

\footnotetext{
${ }^{9} \mathrm{P}$ post-test $=\mathrm{p}$ prétest* ${ }^{*}$ sensibilité/(p prétest*sensibilité $+(1-\mathrm{p} \text { prétest })^{*}(1$-specificité $\left.)\right)$.

${ }^{10} \mathrm{P}$ post-test $=\mathrm{p}$ prétest $*(1-$ sensibilité $) /(\mathrm{p}$ prétest $*(1-$ sensibilité $)+(1-\mathrm{p}$ pretest $) *$ spécificité $)$.

${ }^{11}$ La personne s'autoévalue elle-même, elle est alors son propre juge [59].
} 
FS-Fr variait de 0.15 à 0.40 . Tous ces coefficients de corrélation se trouvaient dans l'intervalle modéré, et les items du BDI-FS-Fr avaient tendance à être modérément corrélés.

Les items les plus fortement corrélés étaient «Sentiments négatifs envers moi-même » et «Attitude critique envers soi » $(\mathrm{r}=0,40)$. Les items les plus faiblement corrélés étaient «Sentiments négatifs envers moi-même » et «perte de plaisir» $(r=0,15)$. La corrélation moyenne inter-items a été calculée pour l'ensemble des items. Cette corrélation doit être comprise entre 0,20 et 0,40 , ce qui suggère que les items du questionnaire sont très homogènes [66]. La corrélation moyenne inter-items du questionnaire du BDI-FS-Fr est de 0.30 ( $\mathrm{p}<0.0001$ ), indiquant une bonne homogénéité des items du BDI-FS-Fr. Ainsi, tous les items du BDI-FS-Fr ont été retenus.

\subsection{Le coefficient de Kaiser-Meyer-Olkin et le test de sphéricité de Bartlett}

Selon Evrard et al. (2003), il convient de vérifier l'adéquation d'échantillonnage, la cohérence des données et la possibilité de leur factorisation [63]. Le test de sphéricité de Bartlett et le test de Kaiser, Meyer et Olkin (KMO) permettent cette vérification. Les items peuvent être factorisables dès que la valeur du KMO dépasse 0.50 [72]. Les résultats montrent qu'un KMO de 0.81 supérieur à 0,50 indique que l'analyse factorielle est possible avec les items du BDI-FS-Fr. Ce résultat est confirmé par le test de Bartlett avec une bonne significativité $(\mathrm{p}<0.001)$. De plus, pour confirmer que tous les items de BDI-FS-Fr ont été retenus, les valeurs de KMO ont été calculées pour chaque item du BDI-FS-Fr. Toutes les valeurs de KMO sont très significatives.

En résumé, l'analyse des items, la corrélation moyenne inter-items, la valeur de KMO, le test de Bartlett et les valeurs de KMO pour chaque item du BDI-FS-Fr confirment le fait que les 7 items de BDI-FS-Fr soient retenus.

\subsection{Analyse factorielle exploratoire (AFE)}

Selon les recommandations d'Evrard et al. (1997), pour une étude exploratoire, l'alpha de Cronbach est acceptable s'il est compris entre 0.6 et 0.8 [63 64]. Pour réaliser l'AFE sur les items du BDI-FS-Fr, l'alpha de Cronbach du BDI-FS-Fr est de 0,74, ce qui est satisfaisant pour une analyse exploratoire. L'analyse factorielle exploratoire avec 7 items des BDI-FS-Fr a été effectuée avec la méthode d'extraction des composantes principales, la règle d'extraction de la courbe des valeurs propres et les critères de Kaiser sur les 473 participants. La règle de Kaiser (1958) [55] (valeur propre supérieure à 1) a servi de base afin de déterminer le nombre de facteurs ${ }^{12}$ à retenir.

Les résultats de l'analyse factorielle exploratoire avec 7 items du BDI-FS-Fr et l'application du critère de Kaiser permettent de retenir un facteur qui représente $40.21 \%$ de la variance totale du BDI-FS-Fr. Ce seul facteur ayant des valeurs propres supérieures à 1 (2.81). La solution à un facteur a été retenue afin d'établir une comparaison avec les items a priori du questionnaire. Après rotation varimax, une concordance a été observée entre les facteurs obtenus à l'aide de l'analyse factorielle et les items du questionnaire (Tableau 5).

Ce facteur explique $40.21 \%$ de l'information avec un KMO de 0,81, il comprend les items $1,2,3,4,5,6$ et 7 , et correspond à la composante cognitive de la dépression et a été nommé «dépression cognitive »; celui-ci explique 40,21 \% de la variance. (Tableau 5). Les saturations ${ }^{13}$ sont importantes à partir de 0,30 . Donc, tous les items ont des saturations élevées sur le facteur 1 .

\footnotetext{
${ }^{12}$ Facteur appelé aussi Composant.

${ }^{13}$ Les coefficients de saturation (factor loading) de la variable sur le facteur considèrent qu'une variable n'est associée à un facteur que si sa saturation est supérieure à 0,30 en valeur absolue. Une valeur de saturation factorielle égale ou supérieure à 0,30 est généralement considérée comme pratiquement significative, c'est-à-dire que cette valeur reflète une saturation adéquate des variables sur le facteur sous-jacent [62].
} 
Tableau 5 : Solution à 1 facteur du BDI-FS-Fr après rotation varimax $(n=473)$.

\begin{tabular}{|l|l|}
\hline Items BDI-FS-Fr & Facteur $\mathbf{1}$ \\
\hline Question 1. Tristesse & $\mathbf{0 , 5 6}$ \\
\hline Question 2. Pessimisme & $\mathbf{0 . 5 7}$ \\
\hline Question 3. Echecs dans le passé & $\mathbf{0 . 5 3}$ \\
\hline Question 4. Perte de plaisir & $\mathbf{0 . 4 4}$ \\
\hline Question 5. Sentiments négatifs envers moi-même & $\mathbf{0 . 6 6}$ \\
\hline Question 6. Attitude critique envers soi & $\mathbf{0 , 4 7}$ \\
\hline Question 7. Pensées ou désirs de suicide & $\mathbf{0 . 6 0}$ \\
\hline \% de la variance expliquée & $\mathbf{4 0 , 2 1 \%}$ \\
\hline Valeurs propres & $\mathbf{2 . 8 1}$ \\
\hline Les coefficients de saturation supérieurs en valeur absolue à 0,30 ont été retenus pour définir les facteurs. \\
Méthode d'extraction : analyse en composantes principales. Méthode de rotation : Varimax. \\
\hline
\end{tabular}

\section{Les variables sexe et âge dans la dépression}

Dans cet échantillon d'étudiants, les femmes ne sont pas plus déprimées que les hommes. Aucun effet significatif n'a été trouvé pour le sexe « Femmes et Hommes » $(\mathrm{t}=1.103$; valeur$\mathrm{p}=0.27)$ et pour l'âge « 16-19 et 20-24 ans » $(\mathrm{t}=-0.9395$; valeur- $\mathrm{p}=0.347)$.

\section{Discussion}

L'objectif principal de cette étude est d'analyser les propriétés psychométriques et de valider l'utilité du BDI-FS-Fr de dépistage de la dépression dans la population étudiante sur un échantillon de 473 étudiants français de $1^{\text {ère }}$ année. La dépression est l'un des troubles psychologiques les plus courants $[68,84]$. Étant donné la prévalence de la dépression dans la population et l'impact au niveau individuel et social de cette maladie, les outils objectifs de mesure de cette dépression sont importants pour une prise en charge thérapeutique et pour un suivi clinique optimum. La présente étude démontre que la version française du questionnaire du BDI-FS-Fr possède des caractéristiques psychométriques adéquates pour permettre son utilisation sur des sujets ayant des troubles dépressifs. La version française du questionnaire BDI-FS-Fr possède des propriétés métrologiques très satisfaisantes pour cette population universitaire. La cohérence interne est bonne avec des coefficients alpha de Cronbach tous supérieurs à 0,70 . La cohérence interne BDI-FS-Fr $(\alpha=0.74)$ est légèrement inférieure à « $\alpha$ » de Beck (alpha dans l'étude originale de Beck égale à 0.86 ; [34]). Dans l'étude de Healey, Kneebone, Carroll, et Anderson en 2008 [73], avec les patients âgés de plus de 65 ans survivants d'un AVC, 1' alpha de Cronbach du BDI-FS était égal à 0.75.

En termes d'applicabilité, le temps de passation prend moins de temps ( \pm 2 minutes) que pour d'autres outils (15 minutes pour le BDI-II et 10 minutes pour l'EDAS-21). Sharp et Lipsky (2002) ont noté que, bien que le temps de réalisation de la BDI-FS soit minime, la sensibilité de la mesure peut être affectée [80]. En outre, le BDI-FS est indiqué pour diagnostiquer de manière différentielle la dépression au sein de la population médicale [24, 79]. Sa réalisation prend moins de 3 minutes et n'est ni une charge pour les étudiants ni pour le personnel à évaluer. En outre il se concentre sur les indicateurs non-somatiques plutôt que sur les symptômes physiques qui pourraient se chevaucher aux plaintes dues à la vie universitaire ou aux événements indésirables associés à la vie. Bien que son utilisation et sa cotation puissent être faciles, le BDI-FS doit être interprété uniquement par des professionnels ayant une formation et une expérience clinique appropriée [24].

Pour la validité convergente et divergente, les corrélations multiples entre les scores d'intensité de dépression et d'anxiété, de stress, et d'estime de soi sont significatives ( $\mathrm{p}<0,001)$. Concernant la validité de critère, les résultats du coefficient de corrélation de 
Spearman (rho) sont significativement corrélés entre le BDI-FS-Fr et les autres échelles (BDIII, EDAS-21 et EDSR) ( $\mathrm{p}<0,001)$. Ainsi, la corrélation, entre le BDI-FS-Fr et les autres échelles qui mesurent la dépression (BDI-II et EDAS-21), nous indique qu'il existe un lien significatif entre les trois mesures $(\mathrm{p}<0,001)$. Lorsque Beck et al. (1997), ont comparé le BDIFS avec une autre mesure standardisée de la dépression, le BDI-FS était positivement corrélé à $0,62, p<0,001$ démontrant ainsi une validité convergente notable [34]. La validité convergente du BDI-FS-Fr confirme l'étude de Beck et al. (1997) [34]. Selon les résultats, le BDI-FS-Fr constitue un test sensible et spécifique pour évaluer la dépression par rapport au BDI-II et l'EDAS-21 (la norme de référence). Les résultats des valeurs prédictives positives (67.07\% et $62.73 \%)$ et des valeurs prédictives négatives $(89.97 \%$ et $87.15 \%)$ sont élevées et démontrent ainsi que le BDI-FS-Fr est fiable. Le BDI-FS-Fr est fortement corrélé au BDI-II, l'EDAS-21 et à l'EDSR. De plus, les résultats du VPP et du VPN montrent que le BDI-FS-Fr constitue un outil de dépistage et aussi de diagnostic de dépression chez l'adulte étudiant. Selon le coefficient Q de Yule, l'intensité de la liaison entre les deux variables BDI-FS-Fr et BDI-II $(0,90)$ et BDI-FS-Fr et EDAS-21 $(0,84)$ est très forte. Enfin, l'efficacité diagnostique de BDI-FS-Fr signifie que les résultats obtenus lors du test sont bons. Dans notre étude, l'AUC du BDI-FS-Fr est supérieure à 0,70. La courbe ROC montre une bonne capacité du BDI-FS-Fr ce qui est compatible avec l'étude de Golden, Conroy, et O'Dwyer, 2007, [75].

Une analyse factorielle exploratoire a dégagé un facteur nommé : « dépression cognitive». Ce facteur explique $40.21 \%$ de la variance totale du BDI-FS-Fr. Cette analyse confirme que le modèle est valide. La validation de l'adaptation française du BDI-FS auprès d'un échantillon de 473 étudiants met en évidence une structure à un facteur qui correspond à celui proposé par Beck et al., en 2000. Dans la présente étude, les résultats ont montré que le BDI-FS-Fr est composé d'un seul facteur. A ce jour, aucune étude ne valide la structure factorielle de la BDIFS en français. La présente étude met en avant la structure à un facteur du BDI-FS dans un échantillon d'étudiants. Cela est cohérent avec des recherches antérieures réalisées par Beck et al., qui ont indiqué une structure à un facteur. Cependant, étant donné le petit nombre d'items de BDI-FS, la probabilité de trouver plus d'un facteur fiable serait extrêmement faible.

Par ailleurs, la passation du BDI-FS-Fr est facile. En d'autres termes, les résultats démontrent que le BDI-FS-Fr mesure bien la dépression. Le BDI-FS-Fr est lié positivement à l'anxiété et au stress, et négativement à l'estime de soi, et il représente bien un concept ayant sa propre spécificité. La fidélité test-retest d'un questionnaire concerne sa reproductibilité, donc sa constance dans la mesure du même phénomène. Les résultats de notre validation de la fidélité test-retest du BDI-FS-Fr montrent un coefficient de corrélation de Pearson de 0,82 ( $\mathrm{p}<0,001)$. Ces résultats sont compatibles avec l'étude de Healey, Kneebone, Carroll, et Anderson en 2008 avec les patients survivants d'un AVC $(0.63, p<0.001)$ [73].

En résumé, la validité convergente et divergente, établie en corrélant les scores du BDI-FSFr et du BDI-II, d'EDAS-21, et d'EDSR, montre que le BDI-FS-Fr a une validité de structure externe très satisfaisante. Concernant la validité discriminante, les sujets dépressifs ont obtenu des scores significativement plus élevés que les participants non- dépressifs ( $p<0,0001)$. De plus, dans notre étude, le BDI-FS-Fr a une sensibilité et une spécificité acceptables avec un score seuil de 4 admis par Beck (2000) comme seuil pathologique. De plus il a une grande efficacité clinique qui est compatible avec l'étude de Steer et al. (1999) réalisée sur les patients ambulatoires (outpatients) [79]. La cohérence interne du questionnaire à 7 items évaluée avec le coefficient alpha de Cronbach est de 0,74 indiquant une très bonne fiabilité du BDI-FS-Fr ; la corrélation moyenne inter-items est à $0,30(\mathrm{p}<0,0001)$; le coefficient de fidélité pair-impair est à $0.73(\mathrm{p}<0,0001)$; le coefficient de fidélité par moitiés (méthode "split-half") est à $0,75(\mathrm{p}<0,0001)$; la fidélité test-retest est de $0,82(\mathrm{p}<0,001)$. L'analyse factorielle indique qu'un seul facteur explique $40,21 \%$ de la variance totale et signifie qu'il a 
une bonne validité de structure interne. Ces résultats confirment l'intérêt d'utiliser le BDI-FSFr pour évaluer la dépression chez les étudiants.

La présente étude montre que le BDI-FS-Fr est un outil de dépistage et aussi de diagnostic de la dépression chez les étudiants. Ce résultat va dans le sens des résultats obtenus dans d'autres pays et sur des populations présentant des pathologies différentes comme les patients infectés par le VIH [74], les patients atteints d'hépatite « $\mathrm{C} »[75]$, les patients survivants d'un AVC [73], les patients souffrant de douleur chronique [76], les femmes en postchirurgie cardiaque [77], et les adolescents «échantillon normatif d'adolescents non médicaux âgés 12-17 ans » [78].

\section{Conclusion}

Le but de cette recherche était de présenter une version française validée du questionnaire du BDI-FS adaptée à la population d'étudiants. Les propriétés psychométriques du BDI-FS-Fr (fiabilité, fidélité et validité) sont très satisfaisantes. Les résultats de cette étude suggèrent que le BDI-FS-Fr est un outil d'évaluation pour les étudiants et peut donc s'avérer utile pour l'évaluation des sujets ayant des troubles dépressifs. Les propriétés et la simplicité d'utilisation de cet outil en font un instrument prometteur de dépistage de la dépression chez les étudiants. Cette étude fournit des preuves solides sur la fiabilité de ce questionnaire rapide de dépistage et de diagnostic de la dépression. Le BDI-FS-Fr est comparable avec le BDI-II et l'EDAS-21 concernant la mesure de la dépression.

Enfin, cette étude est la première qui confirme la validité interne et externe de ce questionnaire et atteste de sa pertinence en langue française.

\section{Remerciements}

Les auteurs tiennent à remercier $\mathrm{M}$. Ataa Allah pour sa participation à l'entrée des données et l'ingénieur statisticien à la plateforme de statistique de la Maison de la Recherche en Sciences Humaines pour ses conseils ainsi que les étudiants pour leur participation à cette étude. 


\section{Références}

1. Gustavsson, A., Svensson, M., Jacobi, F. et al. (2011). Cost of disorders of the brain in Europe 2010, European Neuropsychopharmacology; 21: 718-779.

2. Organisation Mondiale de la Santé. La dépression. Aide-mémoire $\mathrm{N}^{\circ} 369$. Octobre 2012. http://www.who.int/mediacentre/factsheets/fs369/fr/ (consulté le 01.02.2014).

3. Wittchen, H. U., Jacobi, F. (2005). Size and burden of mental disorders in Europe-a critical review and appraisal of 27 studies. European Neuropsychopharmacology; 15:357-376.

4. Kessler, R., Berglund, P., Demler, O. (2005 a). Lifetime Prevalence and Age-of-Onset Distributions of DSM-IV Disorders in the National Comorbidity Survey Replication. Archives of General Psychiatry; 62 : 593-602.

5. Kessler, R., Chiu, W. T., Demler, O. et al. (2005 b). Prevalence, Severity, and Comorbidity of 12-Month DSM-IV Disorders in the National Comorbidity Survey Replication. Archives of General Psychiatry; 62 : 617-627.

6. Keenan, K., Hipwell, A., Feng, X. et al. (2008). Subthreshold Symptoms of Depression in Preadolescent Girls are stable and predictive of Depressive Disorders. Journal of the American Academy of Child and Adolescent Psychiatry; 47(12): 1433-1442.

7. Kessler, R., Avenevoli, S., Merikangas, K. (2001). Mood disorders in children and adolescents: an epidemiologic perspective. Biological Psychiatry; 49 : 1002-1004.

8. Chan Chee C., Beck F., Sapinho D., Guilbert P. (sous la dir.) (2009). La dépression en France - Enquête Anadep 2005, Saint-Denis : INPES, coll. Études santé.

9. Nolen-Hoeksema, S., Hilt, L. (2009). Gender Differences in Depression. In: Gotlieb, I., Hammen (Hrsg.), C. Handbook of Depression. The Guilford Press : New York.

10. Coyne, J. C., \& Calarco, M. M. (1995). Effects of the experience of depression: Application of focus group and survey methologies. Psychiatry: Interpersonal and biological processes, 58, 149-163.

11. Coyne, J. C., Gallo, S. M., Klinkman, M. S., \& Calarco, M. M. (1998). Effects of recent and past major depression and distress on self-concept and coping. Journal of abnormal psychology, 107, 86-96.

12. Bayram, N., Bilgel, N. (2008). The prevalence and socio-demographic correlations of depression, anxiety and stress among a group of university students. Social Psychiatry and Psychiatric Epidemiology 43, 667-672.

13. Shamsuddin, K., Fadzil, F., Salwina, W., Ismail, W., Shah, S.A., Omar, K., Muhammad, N.A., Jaffar, A., Ismail, A., Mahadevan, R. (2013). Correlates of depression, anxiety and stress among Malaysian university students. Asian Journal of Psychiatry, 6: 318323.

14. McDowell, I. (2006). Measuring health: a guide to rating scales and questionnaires. $3^{\mathrm{e}}$ éd. Londres: Oxford University Press; 273-328 et 329-93.

15. Furukawa, TA. (2010). Assessment of mood: Guides for clinicians. J Psychosom Res; 68 (6): 581-9.

16. Gomez, R. (2013). Depression Anxiety Stress Scales: Factor structure and differential item functioning across women and men. Personality and Individual Differences, 54: 687-691.

17. Guelf, J.D., \& Caria, A. (2013). Troubles dépressifs. ${ }^{\circledR}$ PSYCOM, Paris.

18. Jain, S., Carmody, T., Trivedi, M.H., et al. (2007). A psychometric evaluation of the CDRS and MADRS in assessing depressive symptoms in children. J Am Acad Child Adolesc Psychiatry; 46 (9): 1204-12.

19. Phelan, E., Williams, B., Meeker, K., et al. (2010). A study of the diagnostic accuracy of the PHQ-9 in primary care elderly. BMC Fam Pract; 11 (63): 1-9. 
20. Lovibond, P. F., \& Lovibond, S. H. (1995). The structure of negative emotional states: Comparison of the depression anxiety stress scales (DASS) with the Beek depression and anxiety inventories. Behaviour Research and Therapy, 33, 335-343.

21. Beck, A.T., Ward, C.H., Mendelson, M., Mock, J., Erbaugh, J. (1961). An inventory for measuring depression. Arch. Gen. Psychiatry; 4: 561-571.

22. Mohammadian, Y., Shahidi, S,. Mahaki, B., Mohammadi, A.Z., Baghban, A.A., Zayeri, F. (2011). Evaluating the use of poetry to reduce signs of depression, anxiety and stress in Iranian female students. The Arts in Psychotherapy, 38: 59-63.

23. Steer, R. A., Cavalieri, T. A., Lenonard, D. M., \& Beck, A. T. (1999). Use of the Beck Depression Inventory for Primary Care to screen for major depression disorders. General Hospital Psychiatry, 21, 106-111.

24. Beck, A. T., Steer, R. A., \& Brown, G. K. (2000). BDI-FastScreen for medical patients. San Antonio, TX: Pearson.

25. Poole, H., Bramwell, R., \& Murphy, P. (2006). Factor structure of the Beck Depression Inventory-II in patients with chronic pain. Clinical Journal of Pain , 22, 790-798.

26. French translation of the DASS. (2014). Retrieved from http://www2.psy.unsw.edu.au/groups/dass/French/French.htm ; Last updated Nov. 10, 2014.

27. Rosenberg, M. (1965). Society and the adolescent self-image. Princeton, NJ: Princeton University Press.

28. Schmitt, D. P., \& Allik, J. (2005). Simultaneous administration of the Rosenberg SelfEsteem Scale in 53 nations: Exploring the universal and cultural-specific features of global self-esteem. Journal of personality and social psychology, 89, 4, 623-642.

29. Beck, AT, Steer, RA, Brown, GK. (1996). BDI-II Manual. London: The Psychological Corporation.

30. Schonfeld, W. H., Verboncoeur, C. J., Fifer, S. K., Lipschutz, R. C., Lubeck, D. P., \& Buesching, D. P. (1997). The functioning and well-being of patients with unrecognized anxiety disorders and major depressive disorder. Journal of Affective Disorders, 43, 105-119.

31. Shahar, G., \& Davidson, L. (2003). Depressive symptoms erode self-esteem in severe mental illness: A three-wave, cross-lagged study. Journal of consulting and clinical psychology, 71, 890-900,

32. Scheinthal, SM, Steer, RA, Giffin, L, Beck, AT. (2001). Evaluating geriatric medical outpatients with the Beck Depression Inventory-Fastscreen for medical patients. Aging Mental Health 5: 143-148.

33. Silber, E., \& Tippett, J. S. (1965). Self-esteem: clinical assessment ans measurement validation. Psychological reports, 16, 1017-1071.

34. Beck, A. T., Guth, D., Steer, R. A., \& Ball, R. (1997). Screening for depression disorders in medical inpatients with the Beck Depression Inventory for Primary Care. Behaviour Research Therapy, 35, 785-791.

35. Cronbach, LJ. (1951). Coefficient alpha and the internal structure of tests. Psychometrika; 16: 234-97.

36. Feinstein, AR. (1987). Clinimetrics. New Haven and London : Yale University Press.

37. Blascovich, J., \& Tomaka, J. (1991). Measures of self-esteem. In J. P. Robinson, P. R. Shaver, \& L. S. Wrightsman (Eds.), Measures of personality and social psychological attitudes (pp. 115-160). Academic Press, INC: Harcourt Brace Jovanovich, Publishers.

38. Vallières, E. F., \& Vallerand, R. J. (1990). Traduction et validation canadienne-française de l'échelle de l'estime de soi de Rosenberg. International journal of psychology, 25, 305-316. 
39. World Health Report. (2001). Mental health: New understanding. New Hope: World Health Organization.

40. Boyle, GJ. (1994). Does item homogeneity indicate internal consistency or item redundancy in psychometric scales? Person Individ Diff; 12:291-4.

41. Nunnally, J. C. (1978). Assessment of Reliability. In: Psychometric Theory (2nd ed.). New York: McGraw-Hill.

42. Social Psychology Unit- ULB. (2010). Ressources en Statistiques : L'alpha de Cronbach. Source: Stanford University. http://www.psycho-psysoc.site.ulb.ac.be/ressources-enstatistiques/154-lalpha-de-cronbach

43. Smith, S.C., Lamping, D.L., Banerjee, S., Harwood, R., Foley, B., Smith, P, Cook, J.C., Murray, J., Prince, M., Levin, E., Mann, A., \& Knapp, M. (2005). Measurement of health-related quality of life for people with dementia: development of a new instrument (DEMQOL) and an evaluation of current methodology. Health Technology Assessment, 9 (10), 1-112.

44. Nendaz, M.R., Perrier, A.(2004). Sensibilité, spécificité, valeur prédictive positive et valeur prédictive négative d'un test diagnostique. Revue des Maladies Respiratoires, Vol 21, N² -pp. 390-393.

45. Bewick, V., et al. (2004). Statistics review 13: Receiver operating characteristic curves. Critical Care; Vol 8 No 6 (5.8-512).

46. Abbara, Aly. (2015). Biométrie et Organométrie. Paris / France. Dernière mise à jour : Mercredi $18 \quad$ Février, $2015 \quad$ http://www.alyabbara.com/utilitaires/statistiques/khi_carre_rr_odds_ratio_ic.html

47. Perneger, T. \& Perrier, A. (2004). Analyse d'un test diagnostique : courbe ROC, ou « receiver operating characteristic ». Revue des Maladies Respiratoires, Vol 21, № 2 pp. 398-401.

48. Cucherat, M. (2009). Interprétation des essais cliniques pour la pratique médicale. Faculté de Médecine Lyon - Laennec; Mis à jour : aout 2009 http://www.spc.univlyon1.fr/polycop/odds \% 20ratio.htm

49. Nendaz, M.R., Perrier, A.(2004a). Théorème de Bayes et rapports de vraisemblance. Revue des Maladies Respiratoires, Vol 21, Nº 2 -pp. 390-393.

50. Servant, D., Germe, A., Autuori, M., De Almeida, F., Hay, M., Douilliez, C., \& Vaiva, G. (2014). Protocole de relaxation intégrative ettransdiagnostique pour patients anxieux. Résultats d'une étude pilote. L'Encéphale, 40, 501-506.

51. Fombonne, E. \& Fuhrer, R. (1992). «L'application de l'analyse ROC en psychiatrie », Encéphale, XVIT1 : 545-550,

52. Campbell, DT., \& Fiske, DW. (1959). Convergent and discriminant validation by the multitrait-multimethod matrix. Psychological Bulletin, 56(2) : 81 - 105.

53. Hanley, J.A. \& McNeil, J. (1982). The meaning and use of the area under a receiver operating characteristic (ROC) curve. Radiology, 143(1): 29:36.

54. Swets, J.A. (1988). Measuring accuracy of diagnostic systems. Science, 240(4857): 12851293. Tenenhaus, M. (1998). La régression PLS. Technip, Paris.

55. Kaiser, H. F. (1958). The varimax criterion for analityc rotation in factor analysis. Psychometrica, 23 (3), 187-200.

56. Coste, J., Fermanian, J., Venot, A. (1995). Methodological and statistical problems in the construction of composite measurement scales: a survey of six medical and epidemiological journals. Stat. Med. 14: 331-345.

57. Cottraux, J., Bouvard, M., \& Légeron, P. (1985). Méthodes et échelles d'évaluation des comportements. Issy-les-Moulineaux: Ed. d'applications psychotechniques.

58. Bouroche J.M., \& Saporta, G. (1980). L'Analyse des Données, Que sais-je, PUF. 
59. Fermanian, J. (2005). Validation des échelles d'évaluation en médecine physique et de réadaptation : comment apprécier correctement leurs qualités psychométriques. Annales de réadaptation et de médecine physique; 48, 281-287.

60. Lebart, L., Morineau, A. \& Piron, M. (2000). Statistique Exploratoire Multidimensionnelle, Dunod.

61. Delichère, M., \& Memmi, D. (2002). Analyse Factorielle Neuronale pour Documents Textuels. TALN 2002, Nancy.

62. Comrey, A. L., Lee, H. B. (1992). A First Course in Factor Analysis. New York, New jersey : Lawrence Erlbaum Associates.

63. Evrard, Y., Pras, B., et Roux, E. (2003). Market, Etude et recherches en marketing, Ed. DUNOD, Paris.

64. Evrard, Y., Pras, B., et Roux, E. (1997). Market, Etude et recherches en marketing, Paris, Nathan 2è éd.

65. Wasson, J.G., VILlENEUVE, B., MENGIN, N., PELlA, H., et CHANDESRIS, A. (2005). Modèles pressions / impacts, Approche méthodologique, modèles d'extrapolation spatiale et modèles de diagnostic de l'état écologique basés sur les invertébrés en rivière (IBGN). CEMAGREF, Département Gestion des Milieux Aquatiques. Unité de Recherche Biologie des Ecosystèmes Aquatiques. Laboratoire d'Hydroécologie Quantitative, Groupement de Lyon.

66. Piedmont, R.L. (2014). Inter-item Correlations. Encyclopedia of Quality of Life and Well-Being Research, pp 3303-3304.

67. Kubicki, A. Evaluation en Physiothérapie - Numéro 1 - Société de Physiothérapie de Bourgogne.

68. American Psychiatric Association. (2000). Diagnostic and statistical manual of mental disorders: DSM-IV-TR. Washington, DC: American Psychiatric Association.

69. Centre de psychologie appliquée. (1996). Manuel du BDI-II. Paris : Editions du centre de psychologie appliquée.

70. Bouvard, M., et Cottraux, J. (2010). Protocoles et échelles d'évaluation en psychiatrie et psychologie. Editeur: MASSON, 5ème édition. Paris.

71. Martel et al. (2009). Validation de la version française du Questionnaire de Bournemouth. J Can Chiropr Assoc; 53(2).

72. Kaiser, H. F., \& Rice, J. (1974). Little Jiffy, Mark IV. Educational and Psychological Measurement, 34, 111-117.

73. Healey, A. K., Kneebone, I., Carroll, M., \& Anderson, S. J. (2008). A preliminary investigation of the reliability and validity of the Brief Assessment Schedule Depression Cards and the Beck Depression Inventory-Fast Screen to screen for depression in older stroke survivors. International Journal of Geriatric Psychiatry, 23, 531-536.

74. Krefetz, D. G., Steer, R. A., Jermyn, R. T., \& Condoluci, D. V. (2004). Screening HIVinfected patients with chronic pain for anxiety and mood disorders with the Beck Anxiety and Depression Inventory-Fast Screens for Medical Settings. Journal of Clinical Pscyhology in Medical Settings, 11, 283-289.

75. Golden, J., Conroy, R. M., \& O'Dwyer, A. M. (2007). Reliability and validity of the Hospital Anxiety and Depression Scale and the Beck Depression Inventory (full and FastScreen scales) in detecting depression in persons with hepatitis C. Journal of Affective Disorders, 100, 265-269.

76. Poole, H., Bramwell, R., \& Murphy, P. (2009). The utility of the Beck Depression Inventory Fast Screen (BDI-FS) in a pain clinic population. European Journal of Pain, 13, 865-869. 
77. Doering, L. V., Cross, R., Magsarili, M., Howitt, L., \& Cowan, M. J. (2007). Utility of observer-rated and self-report instruments for detecting major depression in women after cardiac surgery: A pilot study. American Journal of Critical Care, 16, 260-269.

78. Winter, L. B., Steer, R. A., Jones-Hicks, L., \& Beck, A. T. (1999). Screening for major depression disorders in adolescent medical outpatients with Beck Depression Inventory for Primary Care. Journal of Adolescent Health, 24, 389-394.

79. Steer, R. A., Cavalieri, T. A., Lenonard, D. M., \& Beck, A. T. (1999). Use of the Beck Depression Inventory for Primary Care to screen for major depression disorders. General Hospital Psychiatry, 21, 106-111.

80. Sharp, L. K., \& Lipsky, M. S. (2002). Screening for depression across the lifespan: A review of measures for use in primary care settings. American Family Physician, 66, 1001-1008.

81. Netemeyer, R. G., Williamson, D. A., Burton, S., Biswas, D., Jindal, S., Landreth, S., Primeaux, S. (2002). Psychometric properties of shortened versions of the Automatic Thoughts Questionnaire. Educational and Psychological Measurement, 62, 111-129.

82. Hollon, S. D., \& Kendall, P. C. (1980). Cognitive self-statements in depression: Development of an automatic thoughts questionnaire. Cognitive Therapy and Research, 4, 383-395.

83. Kendall, P. C., Howard, B. L., \& Hays, R. C. (1989). Self-referent speech and psychopathology: The balance of positive and negative thinking. Cognitive Therapy and Research, 13, 583-598.

84. Koseki, S., Noda, T., et al. (2013). The relationship between positive and negative automatic thought and activity in the prefrontal and temporal cortices: A multichannel near-infrared spectroscopy (NIRS) study. Journal of Affective Disorders $151 ; 352-359$.

85. Central Test. (2009). Validation Psychométrique. 


\section{Annexe 1 : Questionnaire de dépression de Beck (BDI-FS-Fr).}

\section{Choisissez la réponse qui correspond le mieux à votre état au cours de ces $\mathbf{2}$ dernières}

semaines.

\section{Tristesse}

0 - Je ne me sens pas triste.

1 - Je me sens très souvent triste.

2- Je suis tout le temps triste.

3- Je suis si triste ou si malheureux(se), que ce n'est pas supportable.

\section{Pessimisme}

0- Je ne me sens pas découragé(e) face à mon avenir.

1- Je me sens plus découragé(e) qu'avant face à mon avenir.

2- Je ne m'attends pas à ce que les choses s'arrangent pour moi.

3- J'ai le sentiment que mon avenir est sans espoir et qu'il ne peut qu'empirer.

\section{Echecs dans le passé}

0- Je n'ai pas le sentiment d'avoir échoué dans la vie, d'être une(e) raté(e).

1- J'ai échoué plus souvent que je n'aurais dû.

2- Quand je pense à mon passé, je constate un grand nombre d'échecs.

3- J'ai le sentiment d'avoir complètement raté ma vie.

\section{Perte de plaisir}

0- J'éprouve toujours autant de plaisir qu'avant aux choses qui me plaisent.

1- Je n'éprouve pas autant de plaisir aux choses qu'avant.

2- J'éprouve très peu de plaisir aux choses qui me plaisaient habituellement.

3- Je n'éprouve aucun plaisir aux choses qui me plaisaient habituellement.

\section{Sentiments négatifs envers moi-même}

0 - Mes sentiments envers moi-même n'ont pas changé

1- J'ai perdu confiance en moi.

2- Je suis déçu(e) par moi-même.

3- Je ne m'aime pas du tout.

\section{Attitude critique envers soi}

0- Je ne me blâme pas ou ne me critique pas plus que d'habitude.

1- Je suis plus critique envers moi-même que je ne l'étais.

2- Je me reproche tous mes défauts.

3- Je me reproche tous les malheurs qui arrivent.

\section{Pensées ou désirs de suicide}

0 - Je ne pense pas du tout à me suicider.

1- Il m'arrive de penser à me suicider, mais je ne le ferais pas.

2- J'aimerais me suicider.

3- Je me suiciderais si l'occasion se présentait. 